\title{
F-1 - Applicability of Biomarker Analysis in Primary Prevention (through awareness) for Street Runners
}

Gabriela Rosa ${ }^{1}$, Gessyca Pereira de Aquino ${ }^{1}$, Daniel Rogério Petreça ${ }^{1}$, Malu Cristina de Araújo Montoro Lima ${ }^{1}$, Renata Campos ${ }^{1,2}$, Chelin Auswaldt Steclan ${ }^{1}$

${ }^{1}$ Universidade do Contestado, Saúde (SC, Brasil), ${ }^{2}$ Universidade Federal do Paraná, Fisiologia (PR, Brasil)

Support: UnC/Mafra

\begin{abstract}
INTRODUCTION: Street runner is a health-friendly and low-cost practice, which makes it currently biased worldwide. However, this prophylaxis unaccompanied by qualified professionals culminates in a higher incidence of lesions. OBJECTIVES: The aim this study was to evaluate the efficacy of intervention at the primary level (awareness of habits and behaviors that modulate the physiological and biochemistry responses) given by qualified professionals through the analysis of biochemical and physiological parameters, in order to promote health. MATERIALS AND METHODS: The sample had 28 runners (both sexes) with mean age of $32.8 \pm 7.2$, subdivided into control group (without intervention) and test group (submitted to intervention/orientation on modifiable aspects before and after training, for example: feeding/hydration, metabolic/hormonal control, breathing, and posture). Two evaluations were performed, with a 45-day interval in both groups. In each evaluation, the participants were submitted to a 10 minute exercise test (mean intensity), and before and after the test the following parameters were measured: blood glucose and lactate, and spirometry. DISCUSSION AND RESULTS: Spirometric data demonstrated that the airway functionality increased significantly $(p=0.005)$ through the Tiffeneau index only in the test group, which in fact is directly related to the change in the metabolic profile. For the glycemic and lacthemic findings, the test group showed only tendency to maintain and decrease these parameters, respectively. However, even if based, these results demonstrate that there was a beneficial modification of the parameters analyzed after primary intervention. These results reinforce the applicability of the analysis and awareness on biochemical and physiological biomarkers for the promotion of health extrapolating the laboratory and clinical environment. CONCLUSION: Thus, the potential applicability of the intervention by educational processes of awareness is shown here, using basic parameters of biochemistry and physiology as a tool, aiming at the promotion of health at the primary level.
\end{abstract}

Keywords: Health promotion; street runners; biomarkers. 